\title{
Tuberculosis en patrón esporotricoide: una presentación inusual de tuberculosis cutánea en un paciente inmunocompetente
}

\author{
Camila Downey, Lucas Navajas, Romina Andino, Cristian Vera-Kellet y Juan J. Manríquez
}

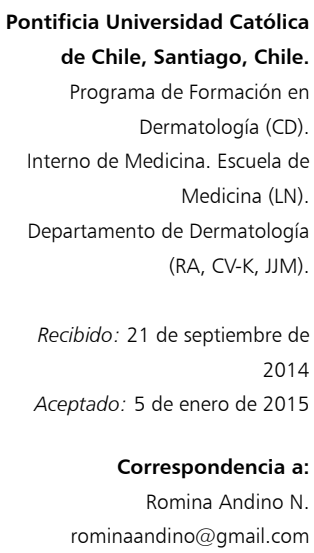

Pontificia Universidad Católica de Chile, Santiago, Chile. Programa de Formación en Dermatología (CD). Interno de Medicina. Escuela de Medicina (LN) Departamento de Dermatología (RA, CV-K, JJM)

Recibido: 21 de septiembre de 2014

Aceptado: 5 de enero de 2015

Correspondencia a: Romina Andino N. rominaandino@gmail.com

\section{Sporotrichoid-like tuberculosis: an unusual presentation of cutaneous tuberculosis in an immunocompetent patient}

Tuberculosis remains a major global health problem. Cutaneous involvement is a rare manifestation of tuberculosis infection. Sporotrichoid clinical pattern consists of a linear arrangement of nodules along the lymphatic vessels. It is often seen in sporotrichosis. Few cases have been reported of cutaneous tuberculosis presenting as a sporotrichoid clinical pattern. We describe a 84-year-old female with ulcerative nodules on upper extremity caused by Mycobacterium tuberculosis, emphasizing the importance of considering cutaneous tuberculosis in the differential diagnosis of sporotrichoid lesions.

Key words: Tuberculosis, cutaneous tuberculosis, Mycobacterium tuberculosis, sporotrichosis.

Palabras clave: Tuberculosis, tuberculosis cutánea, Mycobacterium tuberculosis, esporotricosis.

\section{Introducción}

$\mathrm{E}$ n la actualidad, la tuberculosis sigue siendo un problema de salud pública en el mundo. En el año 2012, 8,6 millones de personas desarrollaron la enfermedad y 1,3 millones murieron a causa de la misma ${ }^{1}$. Para el mismo año, según reportes del Ministerio de Salud de Chile, la prevalencia de tuberculosis en el país era de 13,8 por 100.000 habitantes $^{2}$.

La tuberculosis cutánea es una forma poco común dentro de las presentaciones extrapulmonares, con una prevalencia cercana a $1,5 \%$, entre todos los casos de tuberculosis extrapulmonar ${ }^{3,4,5}$. El agente etiológico es principalmente Mycobacterium tuberculosis, pero en algunos casos se encuentra Mycobacterium bovis, y menos frecuentemente el bacilo de Calmette-Guérin (BCG) ${ }^{6}$.

La infección puede ser adquirida tras la inhalación, ingestión o inoculación del agente, y en la piel es capaz de inducir el desarrollo de granulomas, vasculitis o paniculitis $^{3}$. Las manifestaciones cutáneas de la tuberculosis son múltiples y dependen principalmente de la vía de inoculación (directa, por contigüidad, diseminación hematógena o linfática), la carga de micobacterias y la respuesta inmune del hospedero.

Las lesiones cutáneas de disposición esporotricoide se caracterizan por presentar nódulos con una distribución lineal a lo largo de los vasos linfáticos ${ }^{7}$. Dicho patrón se observa principalmente en la esporotricosis, infección fúngica causada por Sporothrix schenckii, pero también se ha descrito en infecciones por bacterias, hongos y parásitos. Son escasos los casos reportados en la literatura especializada sobre $M$. tuberculosis como agente causal de tuberculosis cutánea con patrón esporotricoide.

\section{Caso clínico}

Paciente de sexo femenino de 84 años, nacionalidad chilena, dueña de casa. Consultó en el policlínico de Dermatología tras presentar un cuadro de dos meses de evolución de nódulos cutáneos eritematosos ulcerados, asintomáticos, en región palmar del dedo meñique derecho y zona ventral de antebrazo derecho, siguiendo un patrón de disposición lineal a lo largo del trayecto de vasos linfáticos (Figura 1); sin otros síntomas acompañantes. La paciente tenía antecedentes de hipertensión arterial esencial, insuficiencia cardíaca congestiva, hipotiroidismo y anemia hemolítica secundaria a crioaglutininas. No presentaba historia previa de tuberculosis, uso de corticoides, inmunosupresión ni trauma reciente en las zonas afectadas. No existían antecedentes familiares de relevancia ni viajes en el último tiempo.

Se planteó el diagnóstico diferencial de micosis subcutánea, micobacteriosis tuberculosa y micobacteriosis atípica, realizándose biopsia y estudio microbiológico de las lesiones cutáneas. El análisis histopatológico reveló una paniculitis granulomatosa supurativa sin caseificación (Figura 2). Las tinciones de PAS, Grocott, Ziehl-Neelsen y Gram no revelaron la presencia de microorganismos en la muestra. Sin embargo, la reacción de polimerasa 
en cadena (RPC) y el cultivo de tejido fueron positivos para $M$. tuberculosis. Dentro el estudio se realizaron hemograma, velocidad de sedimentación, serología para VIH, perfil bioquímico, función renal, análisis de orina, función hepática y radiografía de tórax, los que resultaron todos normales. La determinación de Quantiferon TB gold ${ }^{\circledR}$ resultó positivo. No se realizó PPD.

Basado en los resultados, se estableció el diagnóstico de tuberculosis cutánea y se inició tratamiento con esquema primario de cuatro fármacos: isoniacida, pirazinamida, rifampicina y etambutol. Durante dos meses de seguimiento la paciente continuó con el esquema de terapia antituberculosa de acuerdo a los protocolos establecidos, demostrando una mejoría clínicamente significativa con regresión de las lesiones ulcerativas y sin efectos secundarios reportados.

\section{Discusión}

Chile constituye un país de baja prevalencia de tuberculosis gracias a la existencia del Programa de Control y Eliminación de la Tuberculosis (PROCET), creado hace más de 40 años, y que ha permitido una importante reducción de la morbimortalidad por esta causa. Sin embargo, durante los últimos años hemos sido testigos de un enlentecimiento en el descenso de la incidencia de tuberculosis, la cual pasó de una velocidad de descenso de $8,2 \%$ anual durante la década de los 90 , a sólo $2,8 \%$ anual durante la década 2000-2010 . Según el último informe de la situación de tuberculosis en Chile, la morbilidad total de tuberculosis en todas sus formas, que comprende la suma de casos nuevos y recaídas, fue de 13,6 por 100.000 habitantes durante el año 2013. La incidencia en ese mismo año fue de 12,5 por 100.000 habitantes $^{8}$. Es importante destacar que, del total de casos diagnosticados en 2013, $25 \%$ correspondió a tuberculosis extrapulmonar.

La tuberculosis cutánea ofrece un reto diagnóstico significativo: las manifestaciones son diversas, las lesiones dermatológicas típicas son poco comunes y la identificación del bacilo en tinciones o cultivos es inusual ${ }^{9}$.

La manifestación cutánea puede deberse a diferentes mecanismos patogénicos, ya sea por la infección misma por $M$. tuberculosis o por una reacción inmune a la infección extracutánea por este agente, como también por M. bovis o el bacilo de Calmette-Guérin ${ }^{10}$. La evaluación clínica requiere ser generalmente complementada con exámenes e histopatología. Dentro de las técnicas de tinción destacan: Ziehl-Neelsen, Kinyoun y técnicas fluorocrómicas con auramina-rodamina. La más utilizada es la tinción de Ziehl-Neelsen, requiriendo más de $10^{4}$ bacterias por ml para obtener un diagnóstico positivo, por lo que su utilidad es limitada en muestras paucibacilares $^{11-13}$. El cultivo se considera el estándar de oro para el

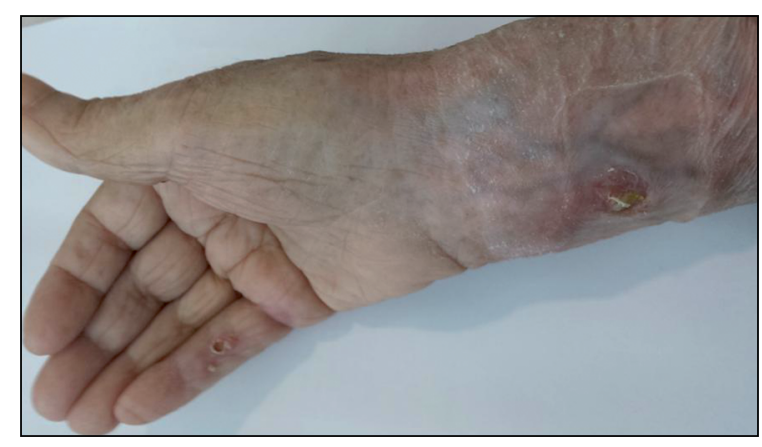

Figura 1. Nódulos ulcerados, exudativos, ubicados en quinta falange, región palmar y antebrazo derecho, siguiendo una distribución lineal paralela al trayecto de los vasos linfáticos.

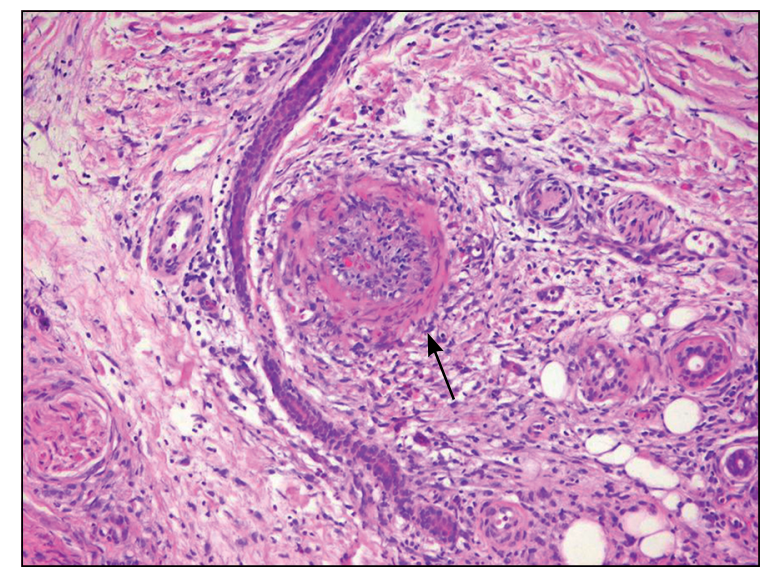

diagnóstico de tuberculosis activa, pero su sensibilidad es significativamente menor en muestras cutáneas 9 .

Los diagnósticos diferenciales de la tuberculosis cutánea son: lesiones por micobacterias atípicas u otras dermatosis infecciosas como sífilis, esporotricosis, cromomicosis, actinomicosis, entre otras ${ }^{14}$. Las manifestaciones clínicas de tuberculosis dependerán de una serie de factores, tales como: la patogenicidad del microorganismo, la resistencia antibacteriana y el estado inmunológico del paciente. Lesiones exógenas adquiridas pueden presentarse como una tuberculosis por inoculación primaria como el chancro tuberculoso, la tuberculosis verrucosa cutis o el lupus vulgar. Por su parte, la diseminación endógena de la infección puede llevar al desarrollo de lupus vulgar, escrofuloderma, tuberculosis miliar, tuberculosis orificial o un absceso tuberculoso metastásico (goma tuberculoso $)^{3}$. Existe otro grupo de manifestaciones conocido como tuberculides, donde el mecanismo patogénico lo constituye una reacción de hipersensibilidad a la infección extracutánea por $M$. tuberculosis, generalmente en individuos inmunocompetentes ${ }^{3}$. Las diversas formas de tuberculides incluyen: eritema indurado de Bazin, liquen escrofulosorum, tuberculide pápulo-necrótica y tuberculide nodular.
Figura 2. Corte histológico obtenido de la lesión que demuestra la presencia de un infiltrado inflamatorio mixto granulomatoso (flecha) en contexto de paniculitis granulomatosa supurativa sin caseificación (H-E, 200X) 
La primera descripción de un patrón esporotricoide como manifestación de una infección cutánea por tuberculosis fue el año 1987 por Premalatha y cols ${ }^{15}$. Posteriormente, han sido escasos los reportes que describen el mismo patrón de disposición morfológica de las lesiones cutáneas por esta infección. La tuberculosis cutánea esporotricoide en estadios precoces suele ser confundida con infecciones estafilocócicas, estreptocócicas, esporotricosis u otras enfermedades granulomatosas ${ }^{4}$. Dada su baja incidencia, existe una falta de conocimiento de esta forma de presentación clínica, lo que se manifiesta en un bajo índice de sospecha inicial como diagnóstico diferencial ${ }^{16-18}$. Dicha afirmación queda de manifiesto en una revisión publicada recientemente donde sólo se encontraron 10 reportes de casos similares ${ }^{19}$.

En el caso presentado se obtuvo un cultivo y un resultado de RPC positivos para M. tuberculosis, respaldando el concepto de que las técnicas de tinción, cultivo y la biología molecular debiesen ser realizadas simultáneamente. En casos de mayor dificultad diagnóstica, incluso se han implementado ensayos terapéuticos con fármacos antituberculosos para lograr la confirmación etiológica ${ }^{20,21}$.

La RPC, así como otras técnicas de biología molecular, ha sido una herramienta útil para detectar y diferenciar M. tuberculosis de otras especies de micobacterias ${ }^{22}$. Un factor limitante es que su sensibilidad se ve reducida significativamente al examinar muestras paucibacilares. En general, la sensibilidad reportada es de $50-72 \%$ en este tipo de ejemplares, con una especificidad que va desde $80-100 \%{ }^{18,20,21,23-27}$. Según Tan y cols, la RPC alcanzaría una sensibilidad de $100 \%$ en tuberculosis cutánea multibacilar; sin embargo, en muestras paucibacilares, como en las formas verrucosa cutis y lupus vulgar, sólo logra detectar $55 \%$ y $60 \%$ de los casos, respectivamente ${ }^{27}$. A su vez, existen estudios que ponen en duda su utilidad, como es el caso de un estudio reciente que reporta cifras bastante menores al evaluar el rendimiento de la RPC en tuberculosis cutánea, siendo cercano a $25 \%{ }^{28}$. El desafío diagnóstico es aún mayor en la era actual donde existe una emergencia de pacientes inmunocomprometidos que se presentan con cuadros clínicos atípicos y que requieren de métodos diagnósticos precisos y oportunos.

El tratamiento de la tuberculosis es gratuito en el sistema público de salud chileno para todos los enfermos del país que lo requieran, independientemente de su pre- visión, nacionalidad o condición migratoria. Todo caso de tuberculosis, ya sea pulmonar o extrapulmonar, tiene indicación de comenzar con el esquema primario de tratamiento, que consta de 50 dosis de isoniacida, rifampicina, pirazinamida y etambutol diario (dos meses), seguido por 48 dosis de isoniazida y rifampicina trisemanal (cuatro meses $)^{29}$. En los casos de tuberculosis extrapulmonar, como en este caso, la norma técnica indica que el inicio del tratamiento debe ser indicado por el especialista que corresponda a la localización de la enfermedad, en coordinación con el neumólogo de referencia del programa. En todos los casos, hay que tener en consideración el tipo de compromiso cutáneo, la etapa de la enfermedad, el nivel de inmunidad y gravedad del paciente ${ }^{30}$.

En suma, la tuberculosis cutánea con patrón esporotricoide es una forma de presentación poco habitual de la infección por $M$. tuberculosis, lo cual refleja la importancia de la sospecha clínica inicial. Pacientes que presenten lesiones con dicho patrón de disposición morfológica, con antecedentes de exposición a este agente, y en países con alta prevalencia de tuberculosis, deberían ser evaluados en forma dirigida para descartar la infección por micobacterias y eventualmente buscar compromiso de otros órganos según el historial clínico. Resulta perentorio realizar un estudio precoz mediante biopsia cutánea, cultivos, tinciones y biología molecular, con el objeto de detectar y tratar oportunamente esta condición de alta relevancia clínica y epidemiológica.

\section{Resumen}

En la actualidad, la tuberculosis sigue siendo un problema de salud pública en el mundo. El compromiso cutáneo es una manifestación poco frecuente de la infección por Mycobacterium tuberculosis. El patrón esporotricoide se refiere a la disposición lineal de los nódulos cutáneos siguiendo el trayecto de los vasos linfáticos, forma de presentación característica de la esporotricosis, de ahí su nombre. Muy pocos casos han sido reportados sobre tuberculosis cutánea con patrón esporotricoide. Se presenta el caso clínico de una mujer de 84 años con nódulos ulcerativos de disposición lineal en miembro superior causados por Mycobacterium tuberculosis, destacando la importancia de incluir a la tuberculosis cutánea en el diagnóstico diferencial de lesiones con patrón esporotricoide.

\section{Referencias bibliográficas}

1.- World Health Organization. Global Tuberculosis Report 2013. Disponible en: http://apps.who.int/ iris/bitstream/10665/91355/1/9789241564656 eng.pd0f. (accedido: 18 de julio de 2014).

2.- Ministerio de Salud, Chile. Tuberculosis: Informe de situación en Chile 2012. Disponible en: http://web.minsal.cl/sites/default/files/files/ tuberculosisInforme2012.pdf (accedido: $18 \mathrm{de}$ julio de 2014).

3.- Lai-Cheong J E, Pérez A, Tang V, Martínez A, Hill V, Menagé Hdu P. Cutaneous manifestations of tuberculosis. Clin Exp Dermatol 2007; 32: 461-6.

4.- Pau W S, AlSaffar H, Weinstein M, Kitai I.
Sporotrichoid-like tuberculosis. Pediatr Infect Dis J 2009; 28: 1135-6.

5.- Pandhi D, Reddy B S, Chowdhary S, Khurana N. Cutaneous tuberculosis in Indian children: the importance of screening for involvement of internal organs. J Eur Acad Dermatol Venereol 2004; 18: 546-51. 
6.- Sehgal V N, Bhattacharya S N, Jain S, Logani K. Cutaneous tuberculosis: the evolving scenario. Int J Dermatol 1994; 33: 97-104.

7.- Göktay F, Aydingöz I E, Mansur A T, Cobanoglu M F, Cavuşoglu C. Detection of Mycobacterium tuberculosis complex by line probe assay in a case with sporotrichoid skin lesions. J Eur Acad Dermatol Venereol 2007; 21: 838-40.

8.- Ministerio de Salud, Chile. Tuberculosis: Informe de situación en Chile 2013. Disponible en: http://web.minsal.cl/sites/default/files/files/ TUBERCULOSIS-INFORME \%202013.pdf (accedido: 15 de enero de 2014).

9.- Almaguer-Chávez J, Ocampo-Candiani J, Rendón A. Current panorama in the diagnosis of cutaneous tuberculosis. Actas Dermosifiliogr 2009; 100: 562-70

10.- Suárez J M, Herrera L, Bautista F J, Dávila J. Skin abscess in a one-year-old infant. Enferm Infecc Microbiol Clin 2004; 22: 361-2.

11.- Sehgal V N, Srivastava G, Khurana V K, Shanma V K, Bhalla P, Beoghar P C. An appraisal of epidemiologic, clinical, bacteriologic, and immunological parameters in cutaneous tuberculosis. Int J Dermatol 1987; 26: 521-6.

12.- Pandhi R K, Bedi T R, Kanwar A J, Bhutani L K. Cutaneous tuberculosis. A clinical and investigative study. Indian J Dermatol 1977; 22: 99-107.

13.- Ramesh V, Misra R S, Jain R K. Secondary tuberculosis of skin. Clinical features and problems in laboratory diagnosis. Int $\mathrm{J}$ Dermatol 1987; 26: 578-81.

14.- Tincopa Wong O W, Sánchez Saldana L. Tuberculosis cutánea. Dermatol Peru 2003; 13 : 195-214
15.- Premalatha S, Rao N R, Somasundaram V, Abdul Razack E M, Muthuswami TC. Tuberculous gumma in sporotrichoid pattern. Int J Dermatol 1987; 26: 600-1.

16.- Remenyik E, Nagy B, Kiss M, Veres I, Sápy M, Horkay I, et al. Sporotrichoid cutaneous Mycobacterium tuberculosis infection in a child. Acta Derm Venereol 2005; 85: 375-6.

17.- Ramesh V. Sporotrichoid cutaneous tuberculosis. Clin Exp Dermatol 2007; 32: 680-2.

18.- Khandpur S, Nanda S, Reddy B S. An unusual episode of lupus vulgaris masquerading as sporotrichosis. Int J Dermatol 2001; 40: 336-9.

19.- Hadj I, Meziane M, Mikou O, Inani K, Harmouch T, Mernissi F Z. Tuberculous gummas with sporotrichoid pattern in a 57-year-old female: A case report and review of the literature. Int J Mycobacteriol 2014; 3: 66-70.

20.- Bothamley G, Batra H, Ramesh V, Chandramui A, Ivanyi J. Serodiagnostic value of the 19 kilodalton antigen of Mycobacterium tuberculosis in Indian patients. Eur J Clin Microbiol Infect Dis 1992; 11: 912-5.

21.- Ramam M, Mittal R, Ramesh V. How soon does cutaneous tuberculosis respond to treatment? Implications for a therapeutic test of diagnosis. Int J Dermatol 2005; 44: 121-4.

22.- Rish J A, Eisenach K D, Cave M D, Reddy M V, Gangadharam P R, Bates J H, et al. Polymerase chain reaction detection of Mycobacterium tuberculosis in formalin-fixed tissue. Am J Respir Crit Care Med 1996; 153: 1419-23.

23.- Welsh O, Vera-Cabrera L, Fernández-Reyes M, Gómez M, Ocampo-Candiani J. Cutaneous tuberculosis confirmed by PCR in three patients with biopsy and culture negative for
Mycobacterium tuberculosis. Int J Dermatol 2007; 46: 734-5.

24.- Ena P, Sechi L A, Saccabusi S, Molicotti P, Lorrai M P, Siddi M, et al. Rapid identification of cutaneous infections by non tubercular mycobacteria by polymerase chain reactionrestriction analysis length polymorphism of the hsp65 gene. Int J Dermatol 2001; 40: 495-9.

25.- Seçkin D, Akpolat T, Ceyhan M, Tuncer S, Turanli A Y. Polymerase chain reaction in cutaneous tuberculosis. Int J Dermatol 1997; 36: 51-4

26.- Margall N, Baselga E, Coll P, Barnadas M A, de Moragas J M, Prats G. Detection of Mycobacterium tuberculosis complex DNA by the polymerase chain reaction for rapid diagnosis of cutaneous tuberculosis. $\mathrm{Br} \mathrm{J}$ Dermatol 1996; 135: 231-6.

27.- Tan S H, Tan H H, Sun Y J, Goh C L. Clinical utility of polymerase chain reaction in the detection of Mycobacterium tuberculosis in different types of cutaneous tuberculosis and tuberculids. Ann Acad Med Singapore 2001; 30 : 3-10

28.- Suthar C, Rana T, Singh U B, Singh M, Ramesh V, Sharma V K, et al. mRNA and DNA PCR tests in cutaneous tuberculosis. Indian J Dermatol Venereol Leprol 2013; 79: 65-9.

29.- Ministerio de Salud, Chile. Normas técnicas para el control y la eliminación de la tuberculosis, 2014. Programa Nacional para el Control y la Eliminación de la Tuberculosis. Disponible en: http://web.minsal.cl/sites/default/ files/NORMA_TECNICA_TUBERCULOSIS. pdf (accedido: 15 de enero de 2015).

30.- Handog E B, Gabriel T G, Pineda R T. Management of cutaneous tuberculosis. Dermatol Ther 2008; 21: 154-61. 